\title{
Sleep and circadian rhythm during a short space mission
}

\author{
A. Gundel ${ }^{1}$, V. Nalishiti ${ }^{2}$, E. Reucher ${ }^{1}$, M. Vejvoda ${ }^{1}$, J. Zulley $^{3}$ \\ ${ }^{1}$ DLR-Institut für Flugmedizin, Köln \\ ${ }^{2}$ ZPK, Star City, Russia \\ ${ }^{3}$ Max-Planck-Institut für Psychiatrie, München
}

Summary. An experiment was conducted to assess sleep and circadian regulation in an orbiting spacecraft. In orbit the weakened influence of 24-h zeitgebers could result in delayed circadian phases with the possibility of a transition to free-running circadian rhythms. This and the specific stressors of a space mission may lead to changes in ultradian sleep regulation and in reduced sleep quantity and quality. During the mission sleep was recorded polygraphically on tape, as was body temperature. Daytime alertness was rated subjectively by a mood questionnaire. For comparison the same parameters were measured during a baseline period preceding the space mission. The circadian rhythms of body temperature and alertness were found to be delayed in space compared to baseline. This may mark a phase shift or the transition to a circadian state of free-run. Sleep was shorter and more disturbed. The structure of sleep was significantly altered. In space REM latency was shorter, there was less REM sleep in the second non-REM/ REM cycle, and slow-wave sleep was redistributed from the first to the second cycle. The self-assessed mood resembled sleep disturbances and adaptation to the space environment. Reduced sleep quality and quantity are likely to result in fatigue and lower daytime performance. Countermeasures should be adopted to improve sleep of astronauts.

Key words: Sleep regulation - Body temperature - Circadian rhythm - Mood - Space mission

In the life of human beings circadian rhythms and sleep are synchronized to the $24-\mathrm{h}$ day determined by the earth's rotation. The synchronization is achieved by the periodicity of bright light, i.e., the change of daylight and darkness within a 24-h period $[3,17]$. Contributions of other zeitgebers to the synchronization are less clear. The circadian

Abbreviations: $\mathrm{EEG}=$ electroencephalogram; $\mathrm{EMG}=$ electromyogram; EOG = electro-oculogram synchronization and the underlying physiological regulation system can be disturbed in many ways. Shift work and traveling across time zones, for example, result in a shift of the perceived day relative to the day in terms of which the circadian system was set. These disturbances have detrimental consequences for sleep and human performance [6, $15]$.

If a spacecraft is not in a geostationary orbit, a natural 24-h light-dark cycle is not present, and in the low orbit of most manned space missions sun rises about every $90 \mathrm{~min}$. This situation should have consequences for circadian and sleep regulation of astronauts. If the light-dark cycle is the most important zeitgeber for the human circadian system a so-called free-run of circadian rhythms may be observed in space as has been found in experiments with human subjects in an isolation unit [16]. The period of these rhythms is longer than $24 \mathrm{~h}$, resulting in a continuous delay of the circadian trough in body temperature and human performance relative to the 24 -h clock. If for operational reasons sleep is not allowed to follow this shift, it becomes desynchronized from the circadian system. This results in changes in REM sleep regulation [18], in sleep disturbances, and in reduced performance during waking hours $[6,15]$.

So far, knowledge about the behavior of human circadian rhythms and sleep under space conditions is more or less anecdotal or has been extrapolated from the situation on earth. This is due to the very limited opportunities for scientists to conduct proper measurements and experiments. Early in the history of manned space flight, Adey et al. [1] obtained sleep recordings from one astronaut for two sleep periods during the Gemini GT-7 flight (see also [10]). The first period was filled by shallow and relatively short sleep; probably as a reaction to this sleep loss, the second period showed a normal sleep structure. On Salyut 6 and 7 body temperature was measured during waking periods and found to be reduced [8]. This reduction was attributed to a decrease in sceletal muscle 
tone. The authors provided no rhythmometric data, but did recommend that a strict work-rest cycle be enforced in space to maintain health and work capacity of cosmonauts (see also [7]). For a stay of longer than 4 months in space, gradual increases in the planned duration of sleep and in total sleep time are reported [8]. From an experiment during the Spacelab 1 mission it was reported [11] that rapid eye movements were increased during the first night in space but were normal the next night. During a prolonged Russian space flight, variations in the percentage of slow-wave sleep were observed in the course of the mission [14]. There is no hypothesis, and no evidence has yet been reported that weightlessness interacts with the regulation of circadian rhythms and sleep.

Not only the lack of a 24-h light-dark cycle or weightlessness could change sleep but also environmental factors. A thorough analysis of self-reported sleep quality and quantity during Shuttle missions has been presented by Santy et al. [13] . According to this study, the average sleep in orbit was more than $1.5 \mathrm{~h}$ shorter than during control periods on earth, where sleep averaged $7.9 \mathrm{~h}$. Astronauts reported their sleep ability to be impaired by several factors including space motion sickness, noise, and excitement. Sleeping drugs were taken at least once by $50 \%$ of the astronauts on dual shifts and by $19.4 \%$ of those on single shift missions. Noticeable sleep disturbances have also been observed in Russian cosmonauts [14].

A recent space mission provided the opportunity to analyze sleep and circadian rhythms during a spaceflight. The data obtained allowed for the first time an assessment of sleep quality, sleep regulation, and circadian regulation in an astronaut who was in space for 8 days. In order to conduct the experiment, special hardware was developed and existing hardware adapted for space applications. With this experiment the above hypotheses on the dynamics of circadian and sleep regulation in an orbiting spacecraft were tested.

\section{Methods}

The astronaut who served the experiment as a subject gave his informed consent to this study, which complied with the recommendations of the Helsinki Declaration. The subject was in space for 8 days. He spent the first 2 days including 2 nights in a launch vehicle before he entered an orbital complex, where he stayed for 6 days and 6 nights before returning to earth. Together with most of the other experimental equipment the hardware for this experiment was brought by an unmanned spacecraft to the spacestation complex several weeks ahead of the mission.

Throughout the mission the subject was requested to rate his mood every hour during waking time. The questionnaire was an adapted and translated version of that of Bond and Lader [2]. It consists of 16 scales representing the three factors of alertness, contendedness, and calmness. For operational reasons the time between two administrations of the mood questionnaire was sometimes longer during mission. After awakening from sleep a sleep questionnaire was given to the subject. It was directed at specifics of the preceding sleep, such as hours of sleep, subjective sleep quality, amount of dreaming, and use of sleep medication. For the first 2 days of the mission this questionnaire was the only source for assessing sleep. Afterwards it complemented polygraphic sleep recordings. Sleep recordings were obtained during nights 3-7 of the mission. Equipment and return items had to be stowed away before the last night. Thus recordings were not possible for the 8th night. Body temperature was measured continuously from day 3 to 7 by means of a rectal probe.

For a comparison of data obtained during the mission baseline measurements were taken for 6 days including 1 night for accommodation to the recording procedures. This baseline experiment was conducted about 8 weeks before the spaceflight. Exactly the same questionnaires and recordings were administered as during the mission.

The mission was conducted under the local time of the training site, and working and sleeping were scheduled accordingly. When the subject spent the days prior to mission close to the launch site, two time zones eastward of the training site, he was also forced to keep his sleep and his meals according to the former local time. Thus there was no shift in time between baseline, premission period and the mission. The training program for the astronaut continued during baseline measurements. He lived in his apartment and left during the day to attend classes and for other activities. Only physical exercise was interrupted in order to avoid an influence on temperature recordings. The subject also did not exercise during mission. As on the orbital complex, where operational constraints determined sleeping times to some extent, the subject was free to choose his sleeping times during baseline. The environmental temperature was not controlled during any period of the experiment.

The principal item of hardware was an Oxford Medilog 8-channel recorder that allows recording body temperature for $24 \mathrm{~h}$ continuously and, in 
addition, sleep polygraphy. The recorder had to be adapted for space application. First, the commercially available connectors and cables for the input signals had to be redesigned. The input cables were replaced by two connectors, one for the temperature probe and the other one for sleep polygraphy, which was recorded only at night. In addition to developing the hardware for a space mission, feasibility of procedures was another important requirement for the equipment. The experiment was to be performed by a single person without any support and within the shortest possible time. To meet these constraints an elastic head band with integrated electrodes was used. Electroencephalogram (EEG) electrodes were placed according to the international $10-20$ system. The electrode positions of this band were $\mathrm{C} 3, \mathrm{C} 4, \mathrm{Cz}$ (ground electrode), and O2. Two electro-oculogram (EOG) signals were derived from the forehead. Electrodes were $\mathrm{Ag} / \mathrm{AgCl}$ electrodes. The band also provided clip-on connectors for four disposable electrodes that served as mastoid references (A1, A2) and as electromyogram (EMG) derivations from the neck. Thus the seven polygraphic channels were C4-A1, C3-A2, O2-A1, EOG1-A2, EOG2-A1, EMG1-A2, and EMG2-A1. This setting provided some useful redundancy.

Another piece of hardware was a device for checking impedances of electrodes and of the temperature probe and for displaying the amplified signals for a quality control. After applying the band and the disposable electrodes the electrodes of the band were filled with a conducting cream. The complete procedure took 10-15 min including improvement of electrode impedances, which were generally less than $5 \mathrm{k} \Omega$. The band was connected to the recorder by a cable with a common electrical shield.

Tapes were brought back to earth and sent to our laboratory in Cologne. In a first step data were analogue/digital converted. The computer allowed a visual scoring of sleep stages in 30 -s periods according to Rechtschaffen and Kales [12]. At the same time signal quality was rated for artifacts and completeness. The rating served as input for subsequent automatic analysis. This included evaluation of the time course of body temperature and of EEG power density. EEG power during sleep is dominated by slow-wave activity. Power density was calculated for the channels $\mathrm{C} 3$ and $\mathrm{C} 4$ and then averaged. Missing values were linearly interpolated. Statistical comparisons of baseline and mission data were conducted by a Wilcoxon test. The mood questionnaire was evaluated by factor analysis. Circadian phase was determined from
Table 1. Daily means and standard errors for nine scales representing the factor alertness and for seven scales representing the factor contendedness/calmness (higher values indicate better mood)

\begin{tabular}{lrr}
\hline & Alertness & \multicolumn{1}{c}{$\begin{array}{l}\text { Contendedness/ } \\
\text { calmness }\end{array}$} \\
\hline Baseline & & \\
Day 1 & $-0.09( \pm 0.11)$ & $0.19( \pm 0.12)$ \\
Day 2 & $-0.06( \pm 0.13)$ & $0.43( \pm 0.09)$ \\
Day 3 & $-0.12( \pm 0.09)$ & $0.19( \pm 0.10)$ \\
Day 4 & $0.19( \pm 0.10)$ & $-0.21( \pm 0.10)$ \\
Day 5 & $-0.95( \pm 0.09)$ & $-0.64( \pm 0.08)$ \\
Mission & & \\
Day 1 & $-0.58( \pm 0.16)$ & $-0.29( \pm 0.15)$ \\
Day 2 & $-0.07( \pm 0.10)$ & $-0.01( \pm 0.10)$ \\
Day 3 & $0.34( \pm 0.12)$ & $0.46( \pm 0.11)$ \\
Day 4 & $0.15( \pm 0.23)$ & $0.39( \pm 0.23)$ \\
Day 5 & $-0.32( \pm 0.14)$ & $-0.42( \pm 0.16)$ \\
Day 6 & $0.79( \pm 0.08)$ & $0.99( \pm 0.08)$ \\
Day 7 & $0.93( \pm 0.08)$ & $1.07( \pm 0.10)$ \\
Day 8 & $0.92( \pm 0.12)$ & $1.11( \pm 0.14)$ \\
\hline
\end{tabular}

the minimum in body temperature after nonparametric regression [5] of values obtained every $30 \mathrm{~s}$.

\section{Results}

Mood ratings were obtained at a total of 128 points in time, 85 ratings during baseline and 43 during mission. The scores of all 16 scales underwent factor analysis (principal components). The analysis of the $16 \times 16$ correlation matrix resulted in two eigenvalues greater than 1 . The corresponding factors represented $60.6 \%$ of the variation in the data. Orthogonal rotation (varimax) of the factor loadings yielded two factors representing $31.0 \%$ and $29.6 \%$ of the variance. One factor loaded on the scales that were expected to measure mainly alertness and the other on those for contendedness and calmness, which could not be separated by the data. No meaningful difference was observed whether data from baseline and mission were analyzed separately or combined.

Table 1 shows daily averages for the scales representing alertness and contendedness/calmness. Higher values represent better mood. The two factors showed very similar time courses. Mood was fairly stable for the baseline period. Only on the last day it was reduced. The first two mission days were rated lower than average baseline. Mood increased during the mission, reaching better mood values toward the end of the mission than during baseline. After the 2 nd night in the orbital complex, however, mood dropped for 1 day. 


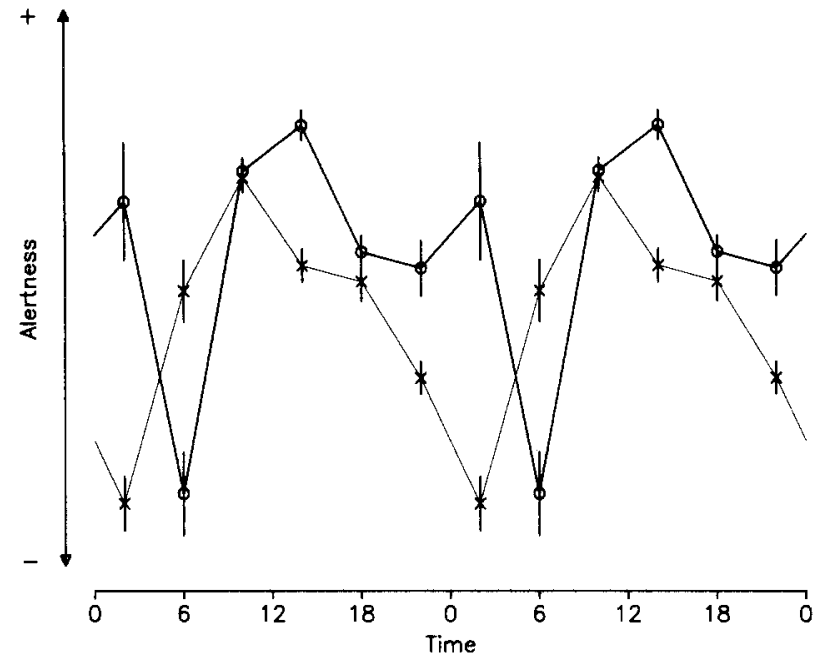

Fig. 1. Average daily alertness and standard errors during baseline and mission. For clarity data are displayed twice. $O$ Mission; $x$ baseline

Separately for baseline and for mission and for both factors, all ratings were averaged that fell into the same 4-h interval starting with the time interval from 0000 to 0400 hours. This yielded clear circadian patterns for both factors, however more markedly for alertness (Fig. 1). The average ratings that are shown at midinterval time 0200 hours were obtained before and those at 0600 hours after sleep throughout. Baseline alertness was lowest before and mission alertness after sleep. Thus they revealed a circadian phase delay from baseline to mission. The other factor also presented a delay.

The five baseline sleep periods, following a night for accommodation to sleeping with electrodes and to being connected to a recorder, are displayed in Fig. 2. Profiles of sleep stages and the time courses of EEG power are presented for each night. EEG power was clearly related to sleep stages, being highest for stages 3 and 4 . The time of sleep onset was very stable and averaged 0029 hours. Mean sleep duration was $6.4 \mathrm{~h}$. Sleep was structured by three to five non-REM/REM cycles. The average number of awakenings per night was 4.6 and the mean awake time during sleep was $6.5 \mathrm{~min}$. As Fig. 2 shows, there was some variability in sleep cycle length and occurrence of slowwave sleep.

Figure 3 shows the corresponding results from mission nights $3-7$. In the first night from which recordings were obtained, both disposable reference electrodes (A1, A2) showed high impedances after about the first sleep cycle, resulting in the loss of polygraphic data for the rest of the night. The end of sleep taken from the subjective sleep report occurred at 0805 hours. Time of sleep onset

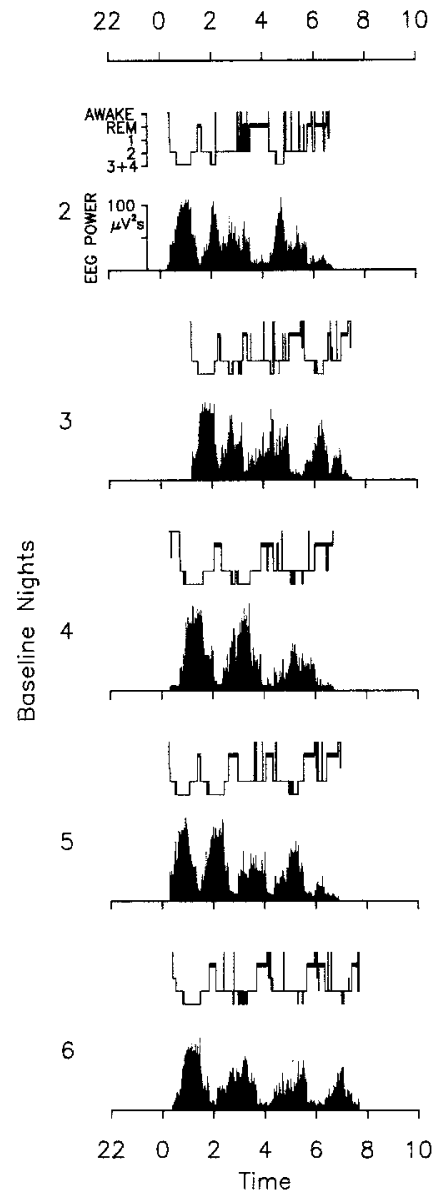

Fig. 2. Sleep analysis for 5 consecutive baseline nights not including an accommodation night. For each night, sleep stages and the distribution of EEG power are shown

varied between 0113 and 0325 , averaging 0151 hours for these five nights. The average sleep duration was $5.63 \mathrm{~h}$. Sleep was comprised of three to four cycles. The number of awakenings from sleep was $7,6,9$, and 0 for nights $4-7$ (average 5.5 awakenings). Times awake for these nights were 10.5 , $33.5,14.0$, and $0 \mathrm{~min}$ (average $14.5 \mathrm{~min}$ per night). Subjective reports showed that sleep quality was lowest in the first two mission nights in the launch vehicle.

A comparison of sleeping times and the amount of sleep is yielded by displaying sleep frequency (Fig. 4). Sleep frequency for mission data is represented by the thick line and that for baseline by the thin line. The hatched area is the contribution of the two sleep periods in the launch vehicle. These data were taken from subjective assessments. The comparison shows a common period of sleep between about 130 and 630 hours except of one instance. Sleep in the launch vehicle was advanced by about $1 \mathrm{~h}$ relative to baseline sleep. Later during the mission sleep was delayed by about $1 \mathrm{~h}$. 


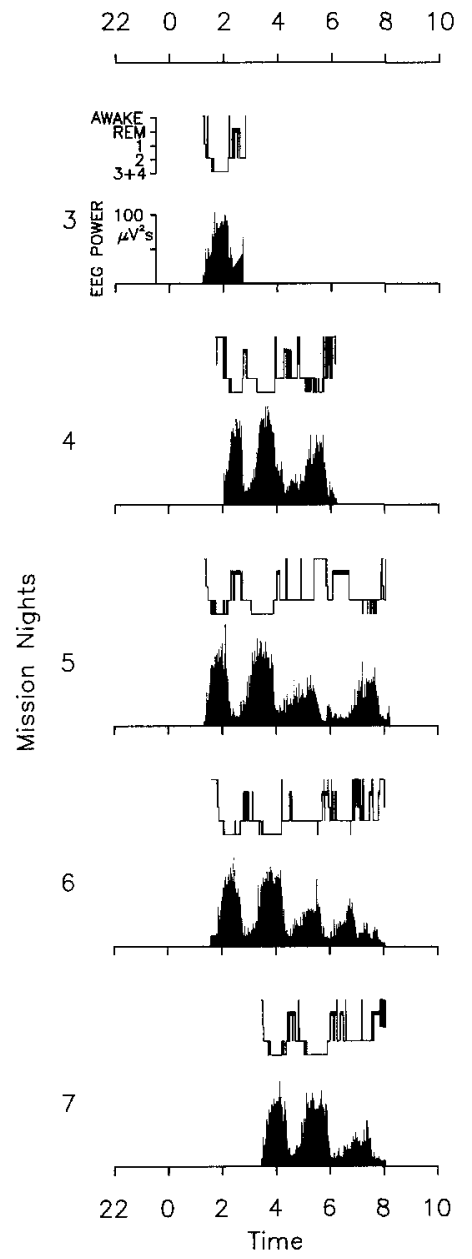

Fig. 3. Sleep analysis for 5 consecutive nights in space. For each night, sleep stages and the distribution of EEG power are shown. In the first recorded night, data could be obtained only for the first sleep cycle

Closer inspection of Figs. 2 and 3 shows that REM latency may have been shorter, and that EEG power in the second cycle may have been higher during the space mission compared to baseline values. Table 2 gives mean values of an analysis of non-REM/REM cycles. Statistical comparisons were performed by a Wilcoxon test. REM latency (the duration of the first non-REM period) was shorter during mission. The duration of the first REM period did not differ, but the second REM period was significantly shorter for mission sleep compared to baseline sleep. Integrated EEG power was significantly lower in the first and higher in the second non-REM period of mission sleep.

By correlation analysis it was checked whether variations in EEG power from night to night could be attributed to a sleep deficit. Neither for baseline nor for the mission was EEG power related to sleep duration in the preceding night or to the time
Table 2. Parameters derived from sleep evaluation described non-REM/REM cycles for baseline nights (averages of 5 nights) and during mission (averages of 4 nights)

\begin{tabular}{lccl}
\hline & Baseline & Mission & Wilcoxon test \\
\hline Duration (min) & & & \\
REM latency & 72.0 & 52.8 & $P<0.05$ \\
Non-REM cycle 2 & 81.3 & 78.5 & \\
Non-REM cycle 3 & 78.0 & 85.0 & \\
REM cycle 1 & 11.0 & 13.3 & \\
REM cycle 2 & 27.3 & 10.0 & $P<0.05$ \\
Integral of power density $\left(\mu \mathrm{V}^{2} \mathrm{~s} \times \mathrm{h}\right)$ & \\
Cycle 1 & 76 & 59 & $P<0.05$ \\
Cycle 2 & 63 & 85 & $P<0.05$ \\
Cycle 3 & 51 & 47 & \\
\hline
\end{tabular}

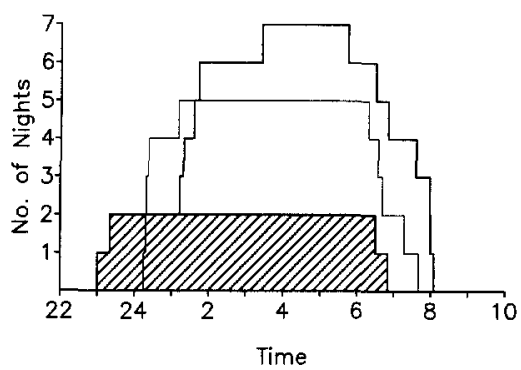

Fig. 4. Sleep frequency during baseline (5 nights, thin line) and during mission (7 nights, thick line). Hatched area, 2 nights in the launch vehicle



Fig. 5. Average daily course of body temperature during baseline (thin line) and during mission (thick line)

awake since the last sleep period. In particular, the shortest night and the longest waking time were not followed by the highest EEG power in the next night. This was true whether the power for the entire sleep or the power for one of the first three sleep cycles were taken. In addition, latency to deep 
sleep (stage 3) and total EEG power did not differ between baseline and mission and was not related to the preceding sleep. The time course of sleep parameters did not indicate an adaptation of mission sleep to baseline values.

Figure 5 shows mean daily body temperature profiles during baseline (thin line) and mission (thick line). The displayed curve is a nonparametric regression of raw data obtained every $30 \mathrm{~s}$. The trough in temperature during baseline occurred at 0554 and at 0737 hours during mission. Average body temperature during sleep was $36.79^{\circ} \mathrm{C}$ for baseline and slightly higher during mission at $36.86^{\circ} \mathrm{C}$.

\section{Discussion}

The results of this experiment present clear evidence for a circadian phase delay during a space mission and an altered structure of non-REM/ REM cycles. The delay was found in the daily minimum of core temperature and in the circadian pattern of self-assessed mood, in particular in the circadian rhythm of subjective alertness.

Regarding the low level of artificial light in a spacecraft and the absence of a 24-h light-dark cycle, a free-run of circadian rhythms with a period longer than $24 \mathrm{~h}$ may be expected. From our data it is not possible to distinguish between a free-run and a phase delay due to weakened zeitgebers. Observations over a longer period of time are necessary for this.

Times for scheduled sleep and meals were the same during the entire experiment although the subject lived in a different time zone $(2 \mathrm{~h}$ eastwards) before launch. If light is the most important zeitgeber, the circadian system of the subject may have advanced prior to launch. This shift would have been in the opposite direction of the observed phase shift during the mission.

Circadian amplitudes were not reduced during the 8-day mission. Again, it is essential to observe amplitudes during a longer mission to draw final conclusions about the stability of circadian rhythms during space flight.

Sleep structure was markedly altered during mission. Adey et al. [1] found it necessary to note that non-REM/REM cycles are still present during a space flight. Quadens and Green [11] observed increased rapid-eye movements for the first night in space. In the present experiment recordings from the first night in space that the subject spent in the launch vehicle were not obtained. Also the observation [14] that the percentage of slow-wave sleep varies during the mission could not be repli- cated. To achieve this, longer observations would be necessary.

It was excluded that sleep deprivation or a sleep deficit was reponsible for our findings of an altered sleep regulation. There was no correlation of prior sleep length or prior waking time and the occurrence of slow-wave sleep. A short REM latency, the dominance of the delta power in the second cycle, and a shorter second REM period characterize the differences to baseline sleep. In particular the finding of a short REM latency together with less REM sleep in the second cycle poses new questions concerning the determinants of sleep regulation in a space environment. There was no adaptation of sleep structure during mission. These findings were unexpected and will be the matter of continuing research.

Severe sleep disturbances due to space motion sickness, noise, or excitement as summarized by Santy et al. [13] according to subjective reports of many astronauts were not observed during this mission. The subject did not experience kinetosis, a probable major cause for sleep disturbances during short flights, nor did he report having taken sleep medication. Undoubtedly, this would indicate difficulties in obtaining or maintaining sleep. However, there were obvious signs of minor sleep disturbances.

These disturbances are expressed by the longer periods of time awake while the subject tried to maintain sleep. These times were longer than during baseline sleep. The interruptions of sleep are reflected by subjective reports and were described as the consequences of thermal discomfort during the first night on the orbital complex and difficulties in the adaptation to the sleeping bag for several nights. The subject reported that abdominal muscle stiffness frequently caused awakenings when he tried to find a comfortable position confined in the sleeping bag. Proper training and information before the mission could probably alleviate these adaptation phenomena and the search for a comfortable sleeping posture and thermal comfort. Since the subject wore foam ear plugs, the noise level in the space station did not cause sleep problems. The observed sleep disturbances are regarded as revealing adaptation problems. They do not belong to the reasons that Litsov and Shevchenko [8] propose enforcing a strict work-rest schedule during space missions.

The short duration of sleep as determined by a late sleep onset due to time demands imposed by the experiments is certainly of greater concern than the observed sleep interruptions. In the 4th and 7 th nights the subject did not obtain more 
than $4.5 \mathrm{~h}$ of sleep. For operational reasons he slept only for about $1 \mathrm{~h}$ in the 8th night, immediately before returning to earth. The consequence of only $4.17 \mathrm{~h}$ sleep in the 4th night is demonstrated by the results of the mood questionnaire on the following day. It shows a distinct drop in alertness, contendedness, and calmness. This decline is most likely accompanied by a decrease in vigilance and performance during day. On this day a drop in performance during a tracking task [9] and an increased midday salivary cortisol concentration [4] were measured. It is our opinion that more time for sleep should be allowed for astronauts who must perform a relatively complicated experimental program.

The daily averages of the mood ratings showed, apart from the drop after the 4th night, a steady improvement of mood during mission starting on board of the launch vehicle, with lower values than during baseline and ending with higher daily averages toward the end of the mission, despite of an obvious sleep deficit after the 7th night. The comparison between baseline and mission may be confounded by an astronaut's professional attitude, aimed at high performance and greatest success.

Average body temperature proved slightly higher during mission compared to baseline values. This could be part of a long-term fluctuation. In addition, it should be noted that the thermal environment was not controlled during the experiment. Therefore, we would not put an emphasis on this difference although it is in contrast to the results of Litsov and Shevchenko [8], who found decreased temperature in space.

Data from a long-duration mission would be necessary to extend the interpretation of this experiment. The results on sleep and circadian regulation during a scientific space mission may also be important for the interpretation of other physiological experiments with results that may depend on prior sleep or on the circadian state, in particular on a phase delay.

\section{References}

1. Adey WA, Kado RT, Walter DO (1967) Computer analysis of EEG data from Gemini flight GT-7. Aerospace Med $38: 345-359$

2. Bond A, Lader M (1974) The use of analogue scales in rating subjective feelings. Br J Med Psychol 47:211-218
3. Czeisler CA, Kronauer RE, Allan JS, Duffy JF, Jewett ME, Brown EN, Ronda JM (1989) Bright light induction of strong (type 0 ) resetting of the human circadian pacemaker. Science 244:1328-1333

4. Drummer C, Heer M, Dressendörfer RA, Strasburger CJ, Gerzer R (1993) Antinatriuretic kidney response to weightlessness. Clin Investig 71:678-686

5. Gasser T, Müller HG, Mammitzsch V (1985) Kernels for nonparametric curve estimation. J Roy Statist Soc B 47:238-252

6. Gundel A, Wegmann HM (1989) Transition between advance and delay responses to eastbound transmeridian flights. Chronobiol Int 6:147-156

7. Litsov AN, Bulyko VI (1983) Principles of organization of rational schedules for crew work and rest during a longterm spaceflight. USSR Report-Space Biol Aerospace Med 17:9-13

8. Litsov AN, Shevchenko VF (1985) Psychophysiological distinctions of organization and regulation of daily cyclograms of crew activities during long-term space-flight. USSR Report-Space Biol Aerospace Med 19:12-18

9. Manzey D, Lorenz B, Schiewe A, Finell G, Thiele G (1993) Beyond physiological aspects of human adaptation to space: analysis of cognitive and psychomotor performance in space during an 8-day mission. Clin Investig 71:725-731

10. Maulsby RL (1966) Electroencephalogram during orbital flight. Aerospace Med 37:1022-1026

11. Quadens O, Green H (1984) Eye movements during sleep in weightlessness. Science $245: 221-222$

12. Rechtschaffen A, Kales A (1968) A Manual of standardized terminology, techniques and scoring system for sleep stages of human subjects. UCLA BIS/BRI, Los Angeles

13. Santy PA, Kapanka H, Davis JR, Stewart DF (1988) Analysis of sleep of shuttle missions. Aviat Space Environ Med 59:1094-1097

14. Stoilova I, Ponomareva IP, Myasnikov VI, Ivancheva $H$, Polyakov VV, Zhukova OP, Peneva N (1990) Study of sleep during a prolonged space flight of the "Mir" orbiting station. In: Boda $\mathrm{K}$ (ed) Current trends in cosmic biology and medicine. Slovak Academy of Sciences, Ivanka Pri Dunaji, pp 85-89

15. Wegmann HM, Gundel A, Naumann M, Samel A, Schwarz E, Vejvoda M (1986) Sleep, sleepiness and circadian rhythmicity in air-crews operating on transatlantic routes. Aviat Space Environ Med 57:B53-B64

16. Wever R (1979) The circadian system of man. Springer, Berlin Heidelberg New York

17. Wever RA, Polasek, J, Wildgruber CM (1983) Bright light affects human circadian rhythms. Pflugers Arch 396:85-87

18. Zulley J (1980) Distribution of REM sleep in entrained 24 hour and free-running sleep-wake cycles. Sleep 2:377-389

Received: December 28, 1992

Accepted: March 15, 1993

Dr. rer. nat. A. Gundel

Institut für Flugmedizin

Deutsche Forschungsanstalt für Luft- und Raumfahrt (DLR)

Linder Höhe

D-51147 Köln, Germany 